\section{Resonance Levels of Neutrons in Silver Nuclei}

THE resonance absorption of neutrons by silver nuclei has been studied with a photo-neutron source by Kurtschatow and Schepkin ${ }^{1}$ and by $\mathrm{us}^{2}$. Continuing this work, we have now estimated the total width of the resonance levels effective for neutron capture which have their energy values lying in the continuous spectrum of the neutrons scattered in a thin paraffin layer. Using the formula of Breit and Wigner ${ }^{3}$ for the capture cross-section as a function of energy, and making some simplifying assumptions, we can evaluate the average spacing between adjacent levels in the energy region concerned, by giving a plausible value to the width of a single level.

In our experiment, the neutron source consists of $50 \mathrm{mgm}$. of radium surrounded with $12 \mathrm{gm}$. of beryllium powder. It gives $1.0 \times 10^{4}$ neutrons per second, as deduced from the work of Chadwick and Goldhaber ${ }^{4}$. In order to produce a continuous spectrum of neutrons, the source is surrounded with a spherical paraffin shell of $0.5 \mathrm{~cm}$. thickness. Two silver detectors of $0.4 \mathrm{~mm}$. thickness are used. Each is screened with cadmium, and subtends a solid angle 0.71 at the source during exposure. According to a formula of Wigner ${ }^{5}$, the neutron-proton scattering cross-section is $11 \times 10^{-24} \mathrm{~cm}^{2}$ for neutrons of $2 \times 10^{5}$ volts. The total number of scattered neutrons received by the detectors is then 450 per second. By means of a Geiger-Müller counter, the number of $\beta$-rays emerging from the surfaces of the silver detectors in the saturation state is found to be $1 \cdot 4$ per second for the activity of half-period 22 sec. We had. measured also the apparent absorption coefficient of the resonance neutrons in a thin silver absorber of $0.020 \mathrm{gm} . / \mathrm{cm} .^{2}$ and get the value $12.5 \times 10^{-22} \mathrm{~cm} .{ }^{2}$ per silver nucleus. For the absorption coefficient of the $\beta$-rays emitted from silver, we find $10 \times 10^{-22} \mathrm{~cm} .^{2}$ per silver atom.

Let us assume that the photo-neutrons consist of one homogeneous group of energy $E_{0}=2 \times 10^{5} \mathrm{e.v}$. ; let $n_{1}$ be the density of the effective resonance levels per volt and $2 \Gamma$ be their half-value width, assumed to be equal for all levels. Then it can be shown by a calculation which will appear elsewhere that the total half-value width summed over the effective resonance levels from zero to $2 \times 10^{5} \mathrm{e} . \mathrm{v}$. can be evaluated from our experimental results. We find $2 \Gamma n_{1} E_{0}=520$ e.v. If $\Gamma$ be given the value $0 \cdot 1-1 \cdot 0$ volt $^{6}$, the average spacing between adjacent effective resonance levels is then of the order $80-800$ volts. This result is in good accord with a recent theoretical estimate given by Bethe $^{7}$, namely, of the order $50-500$ volts. It is also in conformity with the frequency of occurrence of the resonance groups in the low energy region.

Details of this work will be published in the Chinese Journal of Physics.

Physics Department,

$$
\text { C. Y. ChaO. }
$$$$
\text { C. Y. Fu. }
$$

Tsing Hua University, Peiping. Nov. 30 .

\footnotetext{
${ }^{1}$ Kurtschatow and Schepkin, Phys. \%. Soujet., 9, 102 (1936). "Chao and Fu, Tsing Hua Science Rep., A, 3, 451 (1936).

${ }^{3}$ Breit and Wigner, Phys. Rev., 49, 519 (1936).

- Chadwick and Goldhaber, Proc. Roy. Soc., A, 151, 479 (1935).

s See Bethe and Bacher, Rev. Mod. Phys., 8, 117 (1936).

- Frish and Placzek, NATURE, 137, 357 (1936); Preiswerk and Halban, NATURE, 138, 163 (1936).

'Bethe, Phys. Rev., 50, 332 (1936).
}

\section{A New Phenomenon in the Supraconducting Transition of Tantalum and of Tin}

The transition from the supraconducting to the normal resisting state under certain conditions is found not to be continuous but to include a hitherto unsuspected spontaneous increase in resistance followed by a slower decrease. The spontaneous decrease in resistance usually proceeds for several minutes in a succession of steps, until only a fraction of its crest value remains. This crest value of the transient resistance is sometimes 75 per cent of the normal. After such an effect has occurred, it will not occur again when the same conditions are reestablished, unless in the meantime the temperature has been lowered or else a time interval of at least half an hour has elapsed.

These spontaneous changes in resistance can be initiated by a step upward in temperature, in current or in magnetic field, and proceed thereafter as described above while all three external conditions are maintained constant. The combination of temperature, field and current at which this phenomenon appears has been studied and the progress of the resistance change under various conditions noted. The effect is pronounced only when the current is relatively large (about 2 amperes in the tin wire, diameter $0.022 \mathrm{~cm}$.).

A more complete report of this work will appear in the March issue of the Journal of Research of the Nationcl Bureau of Standards.

F. B. Silsbee.

R. B. Sсотт.

F. G. Brickwedde.

National Bureau of Standards, Washington, D.C. Jan. 8.

\section{Influence of Magnetic Fields on Persistent Currents} in Supraconducting Single Crystals of Tin

UP to the present, the determination of the effect of a magnetic field in restoring the resistance in a metal conductor which is in the supraconducting state has depended on the direct measurement of the resistance of the conductor, and consequently it has been difficult to find the exact point at which the first traces of resistance return.

In the McLennan Laboratory at Toronto there have been in use supraconducting galvanometers by means of which it is possible to measure directly, under various conditions, the value of the persistent current in a completely supraconducting circuit. With such an instrument, it is easy to find the magnetic field under which resistance begins to appear, for the smallest resistance causes rapid cessation of the persistent current. These measurements have been carried out for a cylindrical single crystal of tin, in both transverse and longitudinal external fields, at a temperature of $3 \cdot 22^{\circ} \mathrm{K} .\left(0 \cdot 5^{\circ}\right.$ below the normal transition point).

According to the theory of von Laue ${ }^{2}, F$. London ${ }^{3}$, and R. Peierls ${ }^{4}$, the penetration of a transverse magnetic field into a cylindrical supraconductor should commence when the external field has reached one half of the critical field strength, $H_{e}$, and the body should then pass into the so-called 'intermediate state', and show resistance. In a longitudinal field, the resistance should return abruptly when the external field is equal to $H_{c}$.

In Fig. 1 the observed persistent current is plotted against the external magnetic field, in Curve I for 